\title{
Brachial Plexus Blockade
}

Causes Subclinical Neuropathy:

A Prospective Observational Study

\author{
Donato J. Perretta', Matthew Gotlin', Kenneth Brock ${ }^{3}$, \\ Nader Paksima ${ }^{3}$, Michael B. Gottschalk ${ }^{4}$, Germaine Cuff ${ }^{3}$, \\ Michael Rettig ${ }^{3}$, and Arthur Atchabahian ${ }^{3}$
}

\begin{abstract}
Background: The objective of this study is to determine subclinical changes in hand sensation after brachial plexus blocks used for hand surgery procedures. We used Semmes-Weinstein monofilament testing to detect these changes. We hypothesized that patients undergoing brachial plexus nerve blocks would have postoperative subclinical neuropathy detected by monofilament testing when compared with controls. Methods: In total, II 5 hand surgery adult patients were prospectively enrolled in this study. All patients undergoing nerve-related procedures were excluded as well as any patients with preoperative clinically apparent nerve deficits. Eighty-four patients underwent brachial plexus blockade preoperatively, and 31 patients underwent general anesthesia (GA). Semmes-Weinstein monofilament testing of the hand was performed preoperatively on both the operative and nonoperative extremities and postoperatively at a mean of II days on both hands. Preoperative and postoperative monofilament testing scores were compared between the block hand and the nonoperated hand of the same patient, as well as between the block hands and the GA-operated hands. Results: There were no recorded clinically relevant neurologic complications in the block group or GA group. A statistically significant decrease in sensation in postoperative testing in the operated block hand compared with the nonoperated hand was noted. When comparing the operated block hand with the operated GA hand, there was a decrease in postoperative sensation in the operated block hand that did not reach statistical significance. Conclusions: Brachial plexus blockade causes subtle subclinical decreases in sensibility at short-term follow-up, without any clinically relevant manifestations.
\end{abstract}

Keywords: neuropathy, brachial plexus, monofilament, anesthesia

\section{Introduction}

Nerve injury following surgery performed under peripheral nerve blockade may be caused by the block (direct trauma by the needle, local anesthetic or adjuvant neurotoxicity, ischemic injury secondary to pressure and volume of local anesthetic, local inflammatory reaction, and hematoma formation secondary to vascular penetration ${ }^{10}$ ) and surgical factors (surgical tissue damage, tourniquet injury, postoperative swelling, and intraoperative positioning). Prolonged neuropathy after regional anesthesia is fortunately rare, and reported incidence in the literature ranges from $0.04 \%$ to $0.6 \%{ }^{1,5,6}$ However, those studies rely on the reporting by patients of subjective symptoms.

Data suggest that patients with preexisting neuropathy could be more sensitive to nerve injury, ${ }^{4,7,8}$ and the question of whether it is safe to perform nerve blocks in patients with preexisting neuropathy is not settled. ${ }^{9,11,12}$ As a first step in investigating this question, we decided to study patients without preexisting neuropathy following peripheral nerve blocks using Semmes-Weinstein monofilament testing to detect subclinical neuropathy. Semmes-Weinstein monofilament testing is a threshold dependent method of measuring changes in light touch, which is thought to be a very sensitive indicator of neuropathy. ${ }^{2}$ Therefore, it could potentially uncover small sensory deficits that might otherwise not be noticed by the patient or be apparent on gross sensory

\footnotetext{
'Massachusetts General Hospital, Boston, USA

${ }^{2}$ Stony Brook University School of Medicine, NY, USA

${ }^{3}$ New York University School of Medicine, New York City, USA

${ }^{4}$ Emory University School of Medicine, Atlanta, GA, USA

\section{Corresponding Author:}

Donato J. Perretta, Massachusetts General Hospital, 55 Fruit Street,

Yawkey 2, Boston, MA 02। I4, USA.

Email: dperretta@gmail.com
} 
examination. In this study, we measured monofilament sensibility before the brachial plexus nerve block and at the first postoperative visit. Control groups were tested at the same time points and included the nonoperated hand and general anesthesia (GA) cohort. We hypothesized that postoperative monofilament testing on the extremity that received a block would reveal diminished sensation at a rate higher than that of the control groups.

\section{Methods}

The study received Institutional Review Board approval at involved institutions.

Adult patients ( $\geq 18$ years old) undergoing hand surgery procedures at one of our institutions were eligible for enrollment. All surgical procedures were performed at the level of the elbow or distal to it. The decision to use general or regional anesthesia was made by the anesthesiologist and the patient. Ultrasound guidance was used for all blocks. The choice of local anesthetic used for brachial plexus blockade was at the discretion of the attending anesthesiologist. Any patient undergoing a nerve decompression or repair was excluded to eliminate any changes this may have had on sensibility. Surgeries performed using a local anesthetic were also excluded. All patients were prospectively enrolled.

Preoperative monofilament testing was performed on the day of surgery prior to the procedure. We tested 7 areas on both hands using a standardized technique. Examiners were trained in the standardized testing technique. The volar pulps of the thumb, index, middle, and small finger were tested, along with the first dorsal web space. The radial and ulnar aspects of the distal pulp of the ring finger were tested separately. This was done to elucidate whether any particular nerve distributions were more susceptible to sensory changes. The testing technique involved starting with the smallest monofilament and applying perpendicular pressure to the pulp with the monofilament until the monofilament had a slight bend. This was repeated 3 times on each finger with the patient's eyes closed. If the patient was able to recognize sensation 2 out of the 3 times, the patient was deemed to have sensibility to the monofilament. If not, then the next larger sized monofilament was tested.

To facilitate data analysis, a scoring system was devised and applied to each hand. A score was given to each testing zone based on the smallest monofilament that could be felt (Table 1). The score for each testing zone was summed to provide a score for each hand at each time point. Higher scores reflected increased sensation and lower scores decreased sensation. Designations were applied to each hand for labeling purposes, which included the operated hand (block operated) that received the brachial plexus block and the opposite hand that did not receive surgery or the block (block nonoperated). The GA group included the
Table I. Hand Scoring System.

\begin{tabular}{lc}
\hline Monofilament & Points \\
\hline 2.83 & 5 \\
3.61 & 4 \\
4.31 & 3 \\
4.56 & 2 \\
6.64 & 1 \\
\hline
\end{tabular}

Note. This scoring system was used to give each hand a score at each time point. Hands were tested at 7 locations before and after surgery. A hand that was sensate to 2.83 monofilament in each testing location received a score of $35(7 \times 5)$. Lower scores indicate that some testing locations were sensate only to larger monofilaments. For example, if a hand was sensate to the 2.83 monofilament in all zones except the small finger, where it was sensate only to the $3.6 \mathrm{I}$ monofilament, then the hand score would be $34=[(6 \times 5)+(I \times 4)]$. Higher scores indicated higher sensation and lower scores indicated lower sensation.

operated hand (GA operated) that received surgery and the hand that did not receive surgery (GA nonoperated). Preoperative and postoperative scores were compared to evaluate for increases or decreases in sensation. Scores were compared within the block group between the blockoperated and block-nonoperated hands. Changes between the operated and nonoperated hands were compared in the GA group as well. Finally, differences between the blockoperated group and the GA-operated group were compared. The study was powered to detect a difference of 2 in the hand scoring system that was devised.

Statistical analysis was performed using JMP Pro 10 Software (SAS, Inc., Cary, North Carolina). Both parametric and nonparametric tests were used for statistical analysis when the data were normally and nonnormally distributed, respectively. Wilcoxon signed-rank (KruskalWallis) and Mann-Whitney $U$ tests were used for nonparametric tests when continuous variables were analyzed. Chi-square analysis was used for categorical variables for $\mathrm{n}>10$. The Student $t$ test was used for normally distributed continuous variables. A $P$ value of less than .05 was used for statistical significance.

\section{Results}

Demographic data were tabulated and shown in Table 2. There were no complications associated with general or regional anesthesia. The mean age of the patients was similar between the block group and the GA group (46 vs 45). Postoperative monofilament testing was performed at a mean of 10 days postoperatively for the block group and 12 days for the GA group. Within the block group, 62 patients underwent infraclavicular blockade and 22 underwent supraclavicular blockade. Tourniquets were used at least for a portion of the case more than $90 \%$ of the time in both groups. Tourniquet time was inconsistently reported and therefore not included in the 
Table 2. Demographic Data.

\begin{tabular}{lcc}
\hline & Block & General anesthesia \\
\hline Patients & 84 & 31 \\
Mean age & 46 & 45 \\
Mean POD testing performed & 10 & 12 \\
Supraclavicular & 22 & NA \\
Infraclavicular & 62 & NA \\
Tourniquet use & $82(98 \%)$ & $29(94 \%)$ \\
\hline
\end{tabular}

Note. This table outlines the baseline characteristics of the 2 groups. $\mathrm{POD}=$ postoperative day; NA = not applicable.

Table 3. Anesthetic Mixture.

\begin{tabular}{lr}
\hline Anesthetic Mixture & $\mathrm{n}$ \\
\hline Mepivacaine and ropivacaine & 64 \\
Ropivacaine & 12 \\
Bupivacaine & 2 \\
Lidocaine & 1 \\
Lidocaine and ropivacaine & 1 \\
Mepivacaine & 4 \\
Total & 84 \\
\hline
\end{tabular}

Note. This table reports the various anesthetic cocktails used by the anesthesiologist.

Table 4. Surgical Procedures.

\begin{tabular}{lcc}
\hline & Block & General \\
\hline Metacarpal fixation & 12 & 6 \\
Phalanx fixation/ligament repair/tenolysis & 18 & 3 \\
Distal radius ORIF & 11 & 6 \\
Distal ulna resection & 2 & 0 \\
Scaphoid ORIF, S-L repair & 4 & 5 \\
Ganglion/soft tissue mass excision & 7 & 2 \\
Proximal row carpectomy & 1 & 0 \\
Wrist fusion & 1 & 0 \\
Basal joint arthroplasty & 7 & 0 \\
Wrist arthroscopy & 2 & 1 \\
Extensor tendon repair & 5 & 6 \\
M-P ligament repair/reconstruction & 8 & 0 \\
Lateral epicondyle debridement & 2 & 0 \\
Distal biceps repair & 1 & 0 \\
Flexor tendon repair & 1 & 0 \\
Palmar fasciectomy & 2 & 0 \\
Other & 0 & 2 \\
Total & 84 & 31 \\
\hline
\end{tabular}

Note. This table reports the various surgical procedures performed in the 2 groups. ORIF = open reduction and internal fixation.

statistical analysis. The most commonly used medication combination was mepivacaine and ropivacaine (Table 3 ). Table 4 shows the procedures performed in each group. There is a great variety in each group.
Table 5. Operative Hands Average Hand Score.

\begin{tabular}{lrrr}
\hline & Pre-op & Post-op & $\begin{array}{c}\text { Difference pre-op } \\
\text { to post-op }\end{array}$ \\
\hline Block operated & 33.31 & 33.24 & -0.07 \\
GA operated & 32.70 & 33.06 & +0.37 \\
$P$ value & .69 & .60 & .06 \\
\hline
\end{tabular}

Note. This table compares the average hand scores between the block-operated and the GA-operated hands at all time points. No statistical difference was found in preoperative scores between the block-operated and GA-operated hands. The difference between preoperative and postoperative scores trending toward statistical significance $(P=.06)$. Pre-op = preoperative; Post-op = postoperative; $\mathrm{GA}=$ general anesthesia.

Review of the postoperative office charts did not reveal any evidence of persistent clinically evident neuropathy such as sensation or motor deficits, paresthesia, or neuropathic type pain. All patients had at least 2 months of follow-up.

Table 5 shows the average preoperative and postoperative hand scores for the block-operated and GA-operated hands. When comparing the preoperative scores in the GA-operated with the block-operated group, no differences were found $(P=.69)$. Postoperatively, the block-operated group had a small decline in hand scores $(-0.07)$, whereas the GA-operated group had an increase in hand scores $(+0.37)$. This difference did not reach statistical significance $(P=.06)$. We also analyzed the change in scores of each hand from the preoperative to postoperative period as a categorical variable. We compared the percentage of hands that had increased, decreased, or unchanged scores from the preoperative to postoperative period between groups. Table 6 illustrates these results. In all groups, approximately $11 \%$ of patients exhibited increased scores in postoperative testing. In both the block-operated hand and the block-nonoperated hand, scores increased in $11.05 \%$ and $11.22 \%$ of hands, respectively. The GA group closely mirrored this, with $11.52 \%$ of the nonoperated hands and $11.98 \%$ of the operated hands exhibiting increased scores. Differences were seen, however, in the percentages of hand that showed decreased scores postoperatively. The block-operated hand was the only hand in which there were more decreases $(12.59 \%)$ in scores than increases (11.05\%). In the block-operated hand, when comparing preoperative hand scores to postoperative scores, $12.59 \%$ of hands experienced a decreased score. This compared with 7.48\% decreased scores in the block-nonoperated hand. This difference of $5.11 \%$ achieved statistical significance $(P=.01)$ in chi-square analysis. The same analysis was performed in the GA group. In that group, $6.94 \%$ of the operated hands had decreased scores postoperatively compared with $3.23 \%$ of the nonoperated hands. This difference of $3.71 \%$ was not statistically significant $(P=.20)$. In addition, we could not find any nerve or finger distributions more susceptible to decreases in sensibility postoperatively. 
Table 6. Percentage of Hand Scores Increased, Decreased, or Unchanged.

\begin{tabular}{lccc}
\hline & $\begin{array}{c}\text { Increased } \\
(\%)\end{array}$ & $\begin{array}{c}\text { Decreased } \\
(\%)\end{array}$ & $\begin{array}{c}\text { No change } \\
(\%)\end{array}$ \\
\hline Block operated & 11.22 & $12.59^{* * * * * * *}$ & 76.19 \\
Block nonoperated & 11.05 & $7.48^{* *}$ & 81.46 \\
GA operated & 11.98 & $6.91^{* * * * * *}$ & 81.11 \\
GA nonoperated & 11.52 & $3.23^{*}$ & 85.25 \\
\hline
\end{tabular}

Note. This table compares the change in preoperative to postoperative hand scores as a categorical variable (increased, decreased, or no change). We compared the percentages of hands in each group. When comparing the block-operated hands to the block-nonoperated hands, there were significantly more hands with decreased sensation in the block-operated group. In addition, when comparing the block-operated hands to the GA-operated hands, there were more hands with decreased sensation in the block-operated group $(P=.06)$. GA $=$ general anesthesia.

${ }^{*} P=.20 .{ }^{* * P} P=.01 .{ }^{*} * * P=.06$.

Decreased hand scores were also compared between the block-operated hand (12.59\%) and the GA-operated hand (6.94\%). The difference of $5.65 \%$ did not reach statistical significance $(P=.06)$.

Across all variables and time points, the dorsum of the hand demonstrated sensibility to the 2.81 monofilament at a rate of $53 \%$, which was significantly less than the aggregated $80 \%$ rate of the other testing regions $(P<.01)$. This did not vary significantly preoperatively or postoperatively or relative to the block.

\section{Discussion}

The vast majority of literature exploring peripheral nerve block-related neuropathy has used retrospective chart review and questionnaires, which largely rely on selfreported symptoms. For symptoms to be reported, they must exceed a clinically relevant threshold. These symptoms include dysesthesia, paresthesia, and neuropathic pain. ${ }^{6,10}$ The goal of this study was to be more sensitive in detecting neuropathy by using monofilament testing instead of subjective symptoms or gross physical exam. Monofilament testing is commonly used in the plantar aspect of the foot to screen for diabetic peripheral neuropathy and has been shown to be reproducible, albeit with larger sized monofilaments. ${ }^{13}$ It is not routinely used to detect neuropathy after brachial plexus block. We sought to learn the rate of occurrence of these changes and whether they were associated with clinical significant neuropathy. The ideal method of determining this would be to perform blocks in healthy volunteers in the absence of surgery. As this was not feasible, we attempted to appropriately control our study by performing preoperative and postoperative testing on the block nonoperative hand, as well as by including a GA control group.
Preoperatively, no differences were noted in the average hand scores between the block-operated and GA-operated hands, indicating that the groups were similar to begin with. However, we were able to observe that the mean hand score decreased postoperatively $(0.07)$ in the block-operated hand, whereas it increased in the GA-operated hand (0.37). Although this difference did not reach statistical significance $(P=.06)$, the finding of decreased sensation postoperatively in the block-operated hand was corroborated by our analysis using the change in hand score as a categorical variable. The block-operated hand was significantly $(P=.01)$ more likely to have decreased sensation scores postoperatively $(12.59 \%)$ compared with the block-nonoperated hand $(7.48 \%)$. The use of the nonoperated hand serves as a control for the variability that one might expect in monofilament testing. Therefore, the difference between these groups can likely be attributed to something that happened the day of surgery, that is, the block, surgery, or tourniquet. To control for these variables, we looked at our GA group in which surgery under tourniquet was performed in the vast majority of cases, as in the block group. In the control GA group, a statistically significant difference in sensibility preoperation to postoperation was not found when comparing the operated hand $(6.91 \%)$ with the nonoperated hand $(3.23 \%)$. Finding a difference in the block group and not in the GA group suggests that at least some of the difference may be due to the block itself. When comparing the block-operated hand with the GA-operated hand, more blocked hands (12.59\% vs $6.91 \%)$ had decreased postoperative scores, although this did not reach statistical significance either $(P=.06)$. This difference of $5.68 \%$ may represent the true rate of subclinical neuropathy attributable to the block. Thus, it seems that the blocked hand may be more likely to exhibit a decrease in sensitivity to monofilament testing even when controlling for surgery.

The clinical significance of these changes is unknown. None of the block patients in this study had any documented block-associated neuropathy, indicating that the changes may not be clinically relevant. Furthermore, one can see that the magnitude of changes in the block-operated group preoperative to postoperative was very small. Out of a scale of 35, the average decrease in hand score was 0.07 in the block-operated group. Clinically relevant neuropathy in the hand as defined by monofilament testing has not been rigorously defined, but it is unlikely that an average decrease of this amount would be noticeable by the patient or examining physician without the use of monofilaments. The fact that this difference appeared to be subclinical is reassuring, though it might make one more reluctant to perform blocks in patients with preexisting neuropathy to prevent the double-crush phenomenon. Further research is thus needed to determine whether it is 
safe to perform peripheral nerve blocks in patients with preexisting neuropathy.

Interestingly, the nonoperative hands in both the block and GA groups demonstrated small changes when comparing preoperative scores with postoperative scores (Table 6). Increases in sensibility were more common than decreases in these hands. One might attribute variability in monofilament testing as the cause of the differences. However, it is possible that sensory perception is enhanced in the nonoperative hand due to increased sensory awareness after surgery. Also, patients may be more accustomed to the monofilament testing the second time around, allowing them to respond to smaller monofilaments at a higher rate.

The finding of decreased monofilament sensibility at the dorsum of the hand in all testing groups was not expected given the previous reports ${ }^{3}$ that testing is not widely variable across the body. This may be due to the decreased density of sensory end organelles in the nonglabrous skin of the dorsum of the hand compared with distal pulps.

The study has several limitations. There were different examiners administering the monofilament testing at various time points, which could influence the consistency of the data. We attempted to counteract this effect by establishing a standardized testing mechanism. Second, the sample size was much greater in the block group due to the much higher volume of cases performed with this anesthetic technique. Also, the surgical procedures performed in the 2 groups varied and could have had an effect on neuropathy. In addition, we would have liked to perform a multivariate analysis looking at factors contributing to neuropathy such as long tourniquet time. This was not possible given the lack of recorded tourniquet time in the majority of operative reports.

Despite these limitations, this study highlights the fact that brachial plexus blocks can have effects after their intended duration. However, magnitude of differences in sensation found in this study was very small. Therefore, we have not changed our clinical practice and continue to use blocks for the large majority of our hand surgery procedures.

\section{Ethical Approval}

This study was approved by our institutional review board.

\section{Statement of Human and Animal Rights}

All procedures followed were in accordance with the ethical standards of the responsible committee on human experimentation (institutional and national) and with the Helsinki Declaration of 1975, as revised in 2008.

\section{Statement of Informed Consent}

Informed consent was obtained from all patients for being included in the study.

\section{Declaration of Conflicting Interests}

The authors declared no potential conflicts of interest with respect to the research, authorship, and/or publication of this article.

\section{Funding}

The authors received no financial support for the research, authorship, and/or publication of this article.

\section{References}

1. Barrington MJ, Watts SA, Gledhill SR, et al. Preliminary results of the Australasian Regional Anaesthesia Collaboration: a prospective audit of more than 7000 peripheral nerve and plexus blocks for neurologic and other complications. Reg Anesth Pain Med. 2009;34(6):534-541.

2. Bell-Krotoski J. Advances in sensibility evaluation. Hand Clin. 1991;7(3):527-546.

3. Bell-Krotoski J, Weinstein S, Weinstein C. Testing sensibility, including touch-pressure, two-point discrimination, point localization, and vibration. J Hand Ther. 1993;6(2):114-123.

4. Blumenthal S, Borgeat A, Maurer K, et al. Preexisting subclinical neuropathy as a risk factor for nerve injury after continuous ropivacaine administration through a femoral nerve catheter. Anesthesiology. 2006;105(5):1053-1056.

5. Borgeat A, Ekatodramis G, Kalberer F, Benz C. Acute and nonacute complications associated with interscalene block and shoulder surgery: a prospective study. Anesthesiology. 2001;95(4):875-880.

6. Fredrickson MJ, Kilfoyle DH. Neurological complication analysis of 1000 ultrasound guided peripheral nerve blocks for elective orthopaedic surgery: a prospective study. Anaesthesia. 2009;64(8):836-844.

7. Hebl JR, Horlocker TT, Pritchard DJ. Diffuse brachial plexopathy after interscalene blockade in a patient receiving cisplatin chemotherapy: the pharmacologic double crush syndrome. Anesth Analg. 2001;92(1):249-251.

8. Hebl JR, Kopp SL, Schroeder DR, Horlocker TT. Neurologic complications after neuraxial anesthesia or analgesia in patients with preexisting peripheral sensorimotor neuropathy or diabetic polyneuropathy. Anesth Analg. 2006;103(5):1294-1299.

9. Kopp SL, Jacob AK, Hebl JR. Regional anesthesia in patients with preexisting neurologic disease. Reg Anesth Pain Med. 2015;40(5):467-478.

10. Liguori GA. Complications of regional anesthesia: nerve injury and peripheral neural blockade. $J$ Neurosurg Anesthesiol. 2004;16(1):84-86.

11. Lirk P, Birmingham B, Hogan Q. Regional anesthesia in patients with preexisting neuropathy. Int Anesthesiol Clin. 2011;49(4):144-165.

12. Lirk P, Rutten MV, Haller I, et al. Management of the patient with diabetic peripheral neuropathy presenting for peripheral regional anesthesia: a European survey and review of literature. Minerva Anestesiol. 2013;79(9):1039-1048.

13. Valk GD, de Sonnaville JJ, van Houtum WH, et al. The assessment of diabetic polyneuropathy in daily clinical practice: reproducibility and validity of Semmes Weinstein monofilaments examination and clinical neurological examination. Muscle Nerve. 1997;20(1):116-118. 